\title{
Detection of Airway colonization and Invasive Fungal Infections in Cases presenting with Respiratory Diseases in a Tertiary Care Hospital
}

\section{Dr Santwana Verma ${ }^{1}$, Dr Divya Chauhan ${ }^{2}$, Dr Anumeha Gupta ${ }^{3}$, Dr Sunil Sharma ${ }^{4}$, Dr R.S. Negi ${ }^{5}$}

${ }^{1}$ Associate Professor, Dept. of Microbiology, IGMC, Shimla, Himachal Pradesh, India

${ }^{2}$ Senior Resident, Dept. Of Microbiology, IGMC

${ }^{3}$ Junior Resident, Dept. of Microbiology, IGMC, Shimla

${ }^{4}$ Assistant Professor, Dept. Of Pulmonary Medicine, IGMC

${ }^{5}$ Associate Professor, Dept. Of Pulmonary Medicine, IGMC

Corresponding Author

\section{Dr Santwana Verma}

Associate Professor, Dept. of Microbiology, IGMC, Shimla, Himachal Pradesh, India

Email: santwana1812@gmail.com

\begin{abstract}
The respiratory tract is frequently colonized by fungi and bacteria. Candida and Aspergillus species are the common fungi implicated but frequency of different species varies according to geographical areas. Such colonization may progress to invasive fungal disease especially in the immunocompromised individuals.

Aim: To determine fungal pathogens eithercolonizing the airway or causing invasive infection in patients presenting with respiratory symptoms.

Material and Methods: The present study included the historical cohort of 273 cases whose respiratory samples of bronchoalveolar lavage, sputum, endotracheal secretions or pleural fluid, were received for fungal culture between January 2011 and April 2017. The seasonal distribution, demographic profile of age and gender and causative fungi were studied.

Results: Out of 273 cases, 182 were males and 91 females with male to female ratio of 2:1. 60.2\% patients were more than fifty years of age. Out of 273 cases, 161 (58.9\%) showed growth of fungi. Candida was isolated in 93(57.76\%) and Aspergillus in $34(21.11 \%)$ cases. The species of Candida included Candida albicans (46.23\%) followed by C. tropicalis (15.05\%), C. parapsilosis (5.37\%), C. kefyr and C. melibiosica (1.07\% each) and other non-albicans Candida species accounting for 31.18\%. Species of Aspergillus included Aspergillus niger (44.11\%), Aspergillus fumigatus (33.52\%), Aspergillus flavus (11.76\%), Aspergillus nidulans (2.94\%) and other species (17.64\%). Other significant isolates were Penicillum (16), Alternaria (4), Cladosporium (3) and one isolate each of Curvularia lunata, Paeciliomyces lilacinus, Rhizopus arrhizus, Mucor, Geotrichum and Trichosporon species.

Conclusion: Close monitoring of patients with fungal colonization of the respiratory tract may help in early detection of development of invasive fungal disease and institution of antifungal therapy at an early stage when it is most beneficial. Awareness of prevalent species is of epidemiological significance and helps guide antifungal therapy.
\end{abstract}

Keywords: bronchoalveolar lavage, colonization, Candida, Aspergillus 


\section{Introduction}

Fungal infections have emerged as a major public health concern. As the cohort of susceptible hosts is increasing, the prevalence of opportunistic systemic mycoses is escalating. The mycelial fungi like Aspergillus species are ubiquitous in nature and commonly isolated from non-sterile respiratory tract [1]. Yeast like Candida species exist as either commensals or are facultative or obligatory saprophytes $^{[2]}$. The significance of recovering moulds and yeasts from airway is still an unresolved query. The saprophytic colonization of the bronchial tree occurs in patients with pre-existing damage to lung architecture ${ }^{[2]}$. The population having significant immune suppression with haematological and other malignancies, solid organ or bone marrow transplant, therapy with systemic corticosteroids, advanced HIV/AIDS and neutropenia are highly prone to invasive fungal infections. Recent trends have shown considerable level of risk in chronic lung disease like chronic obstructive airway disease (COPD) and recurrent bacterial pneumonias, severe burns or malnourishment, chronic renal and liver failure, liver cirrhosis, post cardiac surgeries, diabetes and tuberculosis. The colonizing fungi may initiate an invasive infection in any of the immune-suppressive conditions. An early detection of colonizing fungi and timely therapeutic intervention may help in preventing life-threatening infections. Thus, bronchoalveolar lavage (BAL), endotrachael aspirate in intensive care unit (ICU) patients, sputum and pleural fluid samples have been studied to assess the airway colonization with fungi or possible invasive fungal infection in patients presenting with respiratory symptoms.

\section{Material Methods}

This study includes the historical cohort of cases whose respiratory samples were received in the Mycology Laboratory between January 2011 and April 2017 over a period of six years and 4 months. A total of 273 samples including 198 bronchoalveolar lavage, 64 sputum, 7 endotracheal aspirates and 4 pleural fluids were studied for fungal isolates. Cytocentrifugation of fluid samples was done at $3000 \mathrm{rpm}$ for 20 minutes in sterile centrifuge tubes ${ }^{[3]}$. Deposits were re-suspended in 1 $\mathrm{ml}$ of supernatant. $10 \% \mathrm{KOH}$ wet mounts were prepared and screened under 100X and 400X magnifications for presence of fungal hyphae, pseudohyphae and budding yeast. Direct microscopy of specimens demonstrating pseudohyphae was correlated with yeasts; septate, hyaline hyphae showing dichotomus branching with Aspergillus sp., broad, irregular, ribbon-like, aseptate hyphae were provisionally considered to be of Zygomycetes and melanised septate hyphae linked to phaeoid fungi. The aliquots of specimen $(100 \mu \mathrm{l} /$ tube $)$ was inoculated in a set of Sabouraud's dextrose agar media with chloramphenicol and incubated at $25^{\circ} \mathrm{C}$ and $37^{\circ} \mathrm{C}$. The culture tubes were examined biweekly up to four weeks. The yeasts were identified by doing Gram staining of smears, sugar fermentation tests of glucose, sucrose, maltose and lactose using $2 \%$ sugar media in tubes with Durham's tubes for detection of gas production, Dalmau's test, and Germ tube test and confirmed using Yeast Panel in BD Phoenix Automated Microbiology System. Moulds were identified morphologically by lactophenol cotton blue wet mount and micro-slide culture for phenotypic characterisation.

\section{Results}

Out of a total of 273 subjects 182 were males and 91 females and the male to female ratio was $2: 1$. Age ranged between 13 years and 90 years. 60.2\% cases were above 50 years of age which includes 39 (24.2\%) between 61 and 70 years, 37 (22.9\%) between 51 and 60 years and $21(13 \%)$ were above 70 years of age (Figure1). Growth of fungi was seen in 161 out of 273 samples constituting 58.9\% isolation rate. Month-wise distribution of moulds is represented in Table 1 . Out of a total of 273 samples, nine, six, 20, 50, 85 and 93 were received successively from 2011 to 2016 (Figure 2). The various fungi isolated included species of Candida (93), Aspergillus (34), Penicillum (16), Alternaria (4), Cladosporium (3), two isolates of family 
hyalohyphomycetes and one isolate each of Curvularia lunata, Paeciliomyces lilacinus, Rhizopus arrhizus, Mucor, Geotrichum and Trichosporon species (Table 2). Majority of the cases showed growth of either Candida species or Aspergillus species (Figure 3-7).

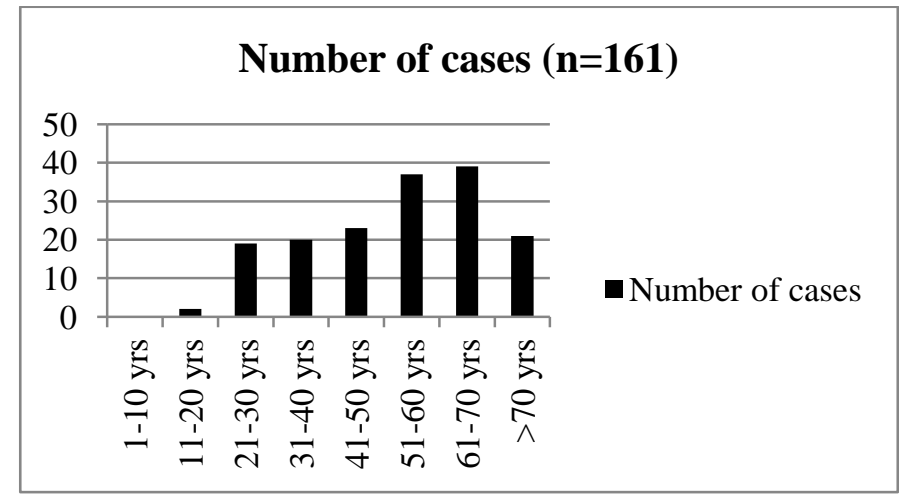

Figure 1 - Age- wise distribution of cases showing invasive disease or airway colonization $(n=161)$

Table 1 Month-wise distribution of important moulds isolated from respiratory samples $(n=53)$

\begin{tabular}{|l|c|c|c|c|}
\hline Month & Aspergillus & $\begin{array}{c}\text { Penicillium \& } \\
\text { Paeciliomyces }\end{array}$ & Zygomycetes & Total \\
\hline Jan & 3 & 1 & 0 & 4 \\
\hline Feb & 1 & 0 & 0 & 1 \\
\hline Mar & 4 & 2 & 0 & 6 \\
\hline Apr & 3 & 1 & 0 & 4 \\
\hline May & 2 & 1 & 1 & 4 \\
\hline Jun & 1 & 0 & 0 & 1 \\
\hline Jul & 4 & 1 & 0 & 5 \\
\hline Aug & 2 & 2 & 0 & 4 \\
\hline Sep & 3 & 3 & 0 & 6 \\
\hline Oct & 3 & 1 & 0 & 4 \\
\hline Nov & 3 & 2 & 0 & 5 \\
\hline Dec & 5 & 3 & 1 & 9 \\
\hline Total & 34 & 17 & 2 & 53 \\
\hline
\end{tabular}

Figure 2 - Distribution of respiratory samples over the study period $2011-2017$ April. $(n=273)$

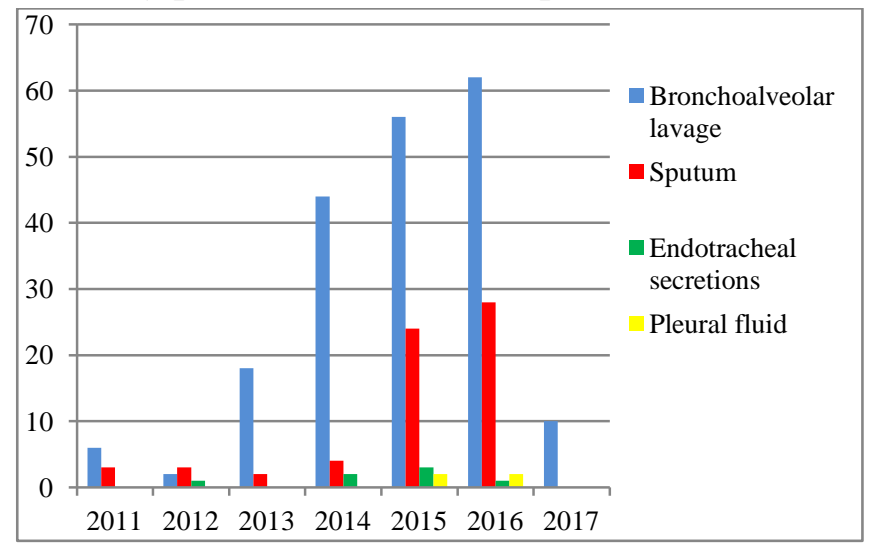

Table 2 - Distribution of fungal isolates according to the samples $(n=161)$

\begin{tabular}{|l|c|c|c|c|c|}
\hline Fungal isolate & BAL & Sputum & ETA & $\begin{array}{c}\text { Pleural } \\
\text { fluid }\end{array}$ & Total \\
\hline Candida albicans & 19 & 22 & 2 & 0 & 43 \\
\hline Candida tropicalis & 12 & 2 & 0 & 0 & 14 \\
\hline Candida parapsilosis & 5 & 0 & 0 & 0 & 5 \\
\hline Candida kefyr & 0 & 1 & 0 & 0 & 1 \\
\hline Candida melibiosica & 1 & 0 & 0 & 0 & 1 \\
\hline $\begin{array}{l}\text { Other non-albicans } \\
\text { Candida }\end{array}$ & 17 & 10 & 2 & 0 & 29 \\
\hline Geotrichum & 0 & 4 & 0 & 0 & 4 \\
\hline Trichosporon & 0 & 1 & 0 & 0 & 1 \\
\hline Aspergillus niger & 14 & 1 & 0 & 0 & 15 \\
\hline Aspergillus fumigatus & 7 & 1 & 0 & 0 & 8 \\
\hline Aspergillus flavus & 4 & 0 & 0 & 0 & 4 \\
\hline Aspergillus nidulans & 0 & 1 & 0 & 0 & 1 \\
\hline $\begin{array}{l}\text { Other Aspergillus } \\
\text { species }\end{array}$ & 5 & 1 & 0 & 0 & 6 \\
\hline Penicillium species & 14 & 2 & 0 & 0 & 16 \\
\hline Alternaria species & 3 & 1 & 0 & 0 & 4 \\
\hline Cladosporium species & 3 & 0 & 0 & 0 & 3 \\
\hline Paeciliomyces lilacinus & 0 & 1 & 0 & 0 & 1 \\
\hline Curvularia lunata & 1 & 0 & 0 & 0 & 1 \\
\hline Rhizopus arrhizus & 0 & 1 & 0 & 0 & 1 \\
\hline Mucor & 1 & 0 & 0 & 0 & 1 \\
\hline Hylohyphomycetes & 1 & 1 & 0 & 0 & 2 \\
\hline Total & 107 & 50 & 4 & 0 & 161 \\
\hline
\end{tabular}

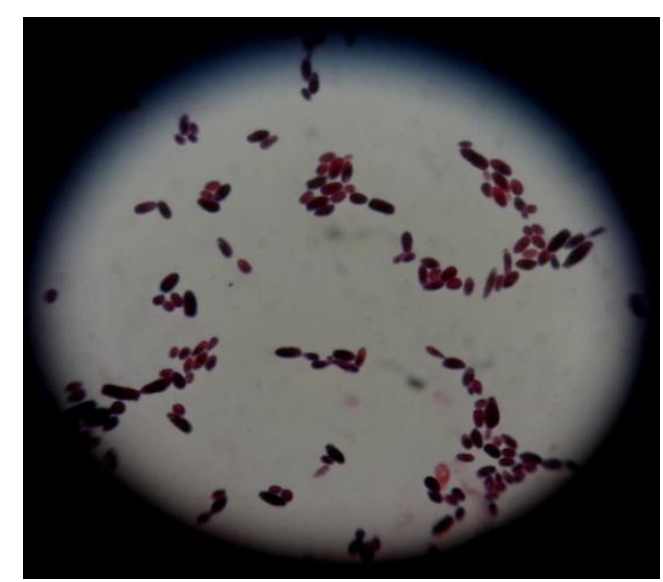

Figure 3 - Gram stained smear of Candida albicans showing budding yeast-like cells (magnification $\mathrm{X} 1000)$

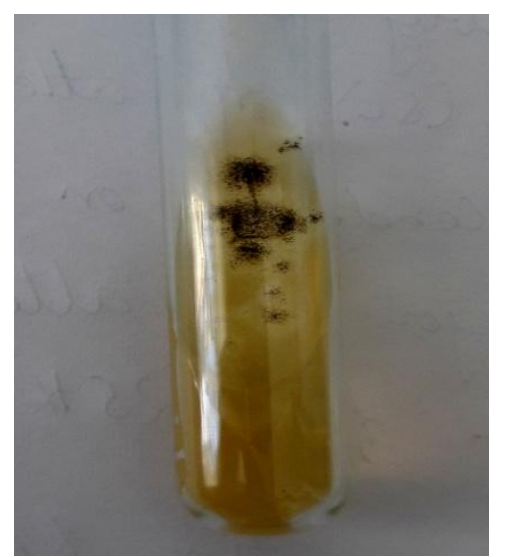

Figure 4 - Sabouraud's dextrose agar culture tube showing powdery black colonies of Aspergillus niger. 


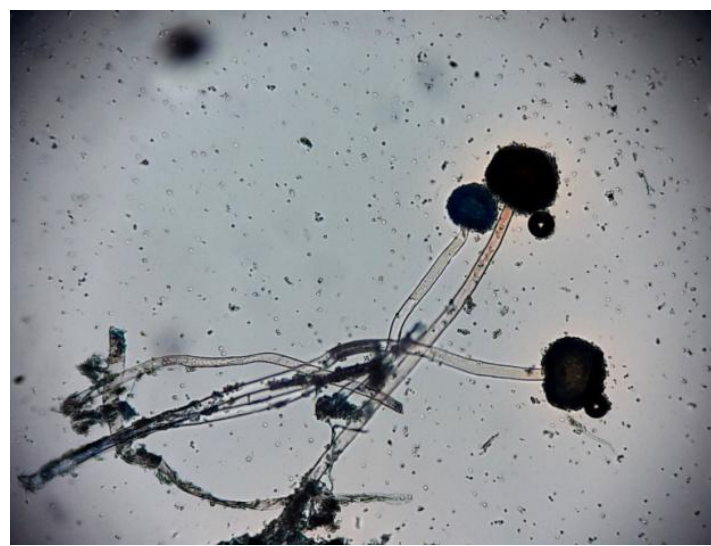

Figure 5 - Lactophenol cotton blue wet mount of Aspergillus niger showing hyaline to brownish conidiophores and black, globose conidial heads with sterigmata and black conidia. (Magnification X 100)

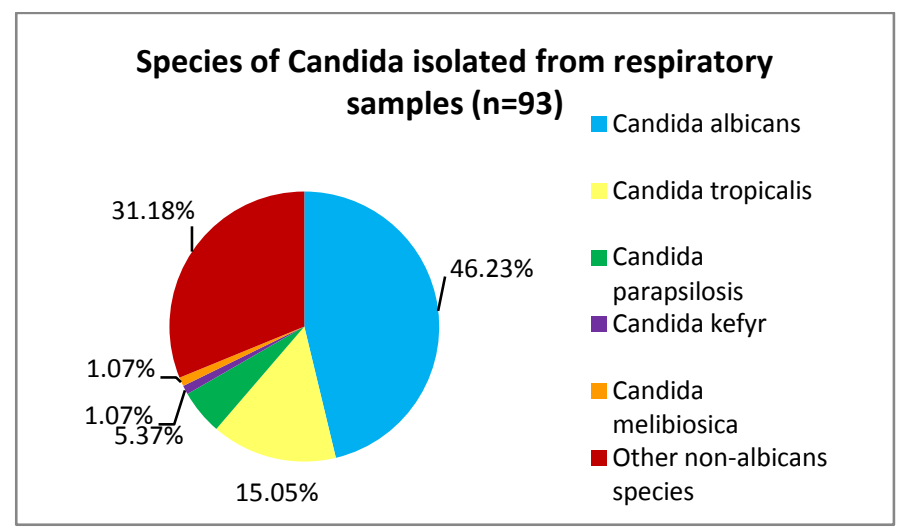

Figure 6 - Species distribution of Candida isolates $(n=93)$

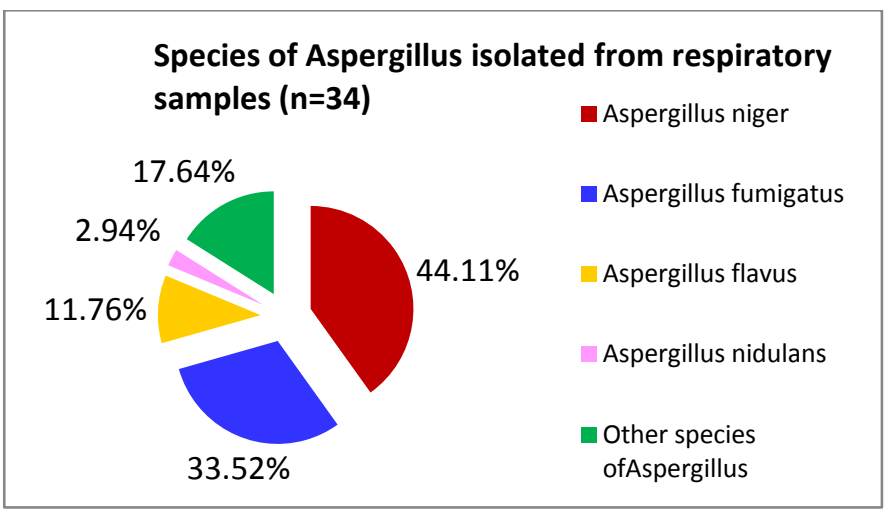

Figure 7 - Species distribution of Aspergillus isolates. $(n=34)$

\section{Discussion}

Respiratory tract infections constitute a major cause of morbidity and mortality more so in the immunocompromised as well as in the immunocompetent individuals. As age advances, the protective mechanisms of the respiratory tract wane and existing lung pathologies provide a suitable nidus for bacteria as well as fungi to colonize. Additionally, co-morbid conditions like diabetes, COPD, and carcinomas are prevalent in elderly. In the present study, more than $60 \%$ of subjects were in their fifth or higher decade of life (Figure 1) Seasonal variations were unremarkable (Table 1) but a significant number of patients were encountered between 2014 and 2017 probably due to improvement in health services and detection rates but also due to awareness amongst the masses (Figure 2). There was a male predominance which can be correlated with the customary habit of smoking compromising the pulmonary function and the fungal infection impinging on already functionally deprived lungs.

The question of colonization versus infection is of critical importance as the tracheobronchial tree is known to be colonized with bacteria and fungi. Aspergillus sp. and Candida sp. are common colonizers although Pneumocystis and Cryptococcus are potential colonizers ${ }^{[4]}$. To exclude arbitrariness, we included only those isolates where culture results were consistent with direct microscopy or all tubes inoculated with the same sample showed growth of same fungus. Those cases which correlated with clinical presentation i.e., aspergilloma, neutropenia, previous or present pulmonary tuberculosis, were included keeping in view that the isolation of Aspergillus in high-risk cases from the respiratory tract is predictive of pulmonary aspergillosis ${ }^{[4]}$. The immunocompetent subjects had significant history of respiratory disorders such as cough, dyspnoea, chest pain, haemoptysis or bronchial asthma.

The colonizing agents may progress to infection which if not detected in time may prove to be fatal. Diagnosis of fungal respiratory infections is always difficult due to lack of pathognomonic clinical features. The ideal specimen for diagnosing invasive fungal disease of respiratory tract are lung biopsies or translaryngeal aspirates but being complicated to obtain are not routinely resorted to [4],[5]. Sputum and bronchoalveolar lavage samples 
are easy to obtain and when collected with due care, contamination with normal flora can be largely prevented. The European Organization for Research and Treatment of Cancer and Mycoses Study Group (EORTC/MSG) have given criteria for proven, probable and possible cases of invasive fungal disease $^{[6]}$. It is difficult to categorise patients according to the EORTC criteria in resource poor setting like ours which is catering to a large population on the one hand and are deficient of infrastructure and facilities on the other.

Candida sp. and Aspergillus sp. constitute the bulk of fungi reported in pulmonary mycoses. Their relative proportion and species distribution have shown considerable geographical variation. The two leading causative fungi were Candida sp. accounting for $57.76 \%$ and Aspergillus sp. isolated in $21.11 \%$ of our cases (Table 2) (Figures 3,4,5). The incidence of Candida pneumonia ranges between $0.23 \%$ and $8 \%{ }^{[3],[4]}$. The most common species in our study was Candida albicans (46.23\%) followed by C. tropicalis (15.05\%), C. parapsilosis (5.37\%), C. kefyr and C. melibiosica (1.07\% each) and other non-albicans Candida species which could not be identified up to species level constituted $31.18 \%$ (Figure 6). Zarrinfar et al have reported similar results with $52 \%$ C. albicans, $24 \%$ C. tropicalis and $1.3 \%$ each $C$. kefyr and $C$. parapsilosis ${ }^{[3]}$. But in contrast, $C$. glabrata, $C$. krusei and $C$. guilliermondii were also recovered from $14.7 \%, 5.3 \%$ and $1.3 \%$ cases respectively in that study ${ }^{[3]}$. In another study from the Himalayan region, Candida albicans and $C$. tropicalis constituted the most frequently isolated species of Candida $^{[5]}$. Our results are also similar to those of Montero et al ${ }^{[7]}$.

The Aspergillus sp. may colonize the respiratory tract and in patients with neutropenia, COPD, solid organ transplant or other chronic lung pathologies may subsequently develop invasive fungal disease. Among immunocompromised patients, mortality is as high as $92 \%{ }^{[4],[7]}$. The various species isolated in the present study are Aspergillus niger (44.11\%), Aspergillus fumigatus (33.52\%), Aspergillus flavus (11.76\%), Aspergillus nidulans (2.94\%) and other species (17.64\%) (Figure 7). There are epidemiological differences across the globe. Most medical literature documents Aspergillus fumigatus as the leading cause of aspergillosis. An incidence of $80-90 \%$ has been reported in critically ill patients from Spain with recent increase in Aspergillus flavus and Aspergillus terreus ${ }^{[7]}$. Fungal culture of BAL samples in a study by Biswas et al yielded $A$. flavus (21.4\%), A. fumigatus (14.3\%) and A. niger $(10.7 \%){ }^{[5]}$ Contrary to this Aspergillus niger was the leading isolate in the present study. It is considered to be an unusual cause of invasive pulmonary aspergillosis ${ }^{[8]}$. A. niger is more often associated with otomycosis and in cases where it was obtained from pulmonary specimens, the outcome has been fatal with $75 \%$ mortality rate ${ }^{[9]}$. Thus, the finding of Aspergillus niger as the most prevalent species in our region is of great significance as such cases would need intensive management. The limitation of our compilation is that the patients from whom Aspergillus niger was recovered could not be followed up to final outcome. Mucormycosis caused by opportunistic fungi of the Zygomycetes class and genera Rhizopus, Mucor and Lichthemia are rarely reported ${ }^{[7]}$. Two of our subjects showed growth of Rhizopus arrhizus and Mucor sp. Other unusual fungal agents included yeasts; Geotrichum and Trichosporon and moulds Paeciliomyces lilacinus, Curvularia lunata, Penicillium sp., Cladosporium sp., and Alternaria sp. Since geographical variation is a major consideration in changing epidemiology, there is a need to conduct research to study changing patterns. These isolates have been reported as unusual agents of invasive fungal disease from different parts of the world $^{[2]}$.

Close monitoring of patients with fungal colonization of the respiratory tract may help in early detection of development of invasive fungal disease and institution of antifungal therapy at an early stage when it is most beneficial. This would help curb the high incidence of mortality as no guidelines are as yet available and significance of recovering moulds and yeasts from airway is still doubtful. The knowledge of fungi inhabiting the 
respiratory tract is also of epidemiological significance to foresee an impending invasive fungal disease.

\section{References}

1. A. Chakrabarti, Mould infection in ICUs. SIHAM- $11^{\text {th }}$ National Conference of Society of Indian Human and Animal Mycologists, 2016.

2. K.J.Kwon-Chung and J.E. Bennett, Aspergillosis, . Lea and Febiger, Philadelphia, United States Of America: Medical Mycology, 1992.

3. H. Zarrinfar, s. Kaboli, S. Dolatabadi, and R. Mohammadi, "Rapid detection of Candida species in bronchoalveolar lavage fluid from patients with pulmonary symptoms," Brazilian Journal of Microbiology,vol. 47, pp. 172-176, 2016.

4. K.S. Knox and L. Meinke, "Role of bronchoalveolar lavage diagnostics in fungal infections," Clin Chest Med, vol. 30, pp.355-365, 2009.

5. D. Biswas, S. Agarwal, G. Sindhwani and J. Rawat, "Fungal colonization in patients with chronic respiratory diseases from Himalayan region of India," Annals of Clinical Microbiology and Antimicrobials, vol. 9, pp. 28-34, 2010.

6. B.D. Pauw, T.J. Walsh, J. Donnelly, D.A. Stewns, J.E. Edwards, T. Calandra, et al, " Revised definitions of Invasive Fungal Disease from the European Organization for Research and Treatment of Cancer/ Invasive," Clin Infect Dis, vol. 46(12),pp.1813-1821, 2008.

7. J.G.Montero, P. Olaechea, F.A.Lerma, L.A.Rocha, J. Blanquer, B. Galvan, A. Rodriguez, et al, "Epidemiology, diagnosis and treatment of fungal respiratory infections in the critically ill patient," Rev Esp Quimioter, vol.26(2), pp. 173-188, 2013.

8. A.K.Person, "Aspergillus niger: an unusual cause of invasive pulmonary aspergillosis," J Med Microbiol, vol. 59, pp. 834-838, 2010.
9. L. Fianchi, M. Picardi, L. Cudillo, L. Corvatta, L. Mele, G. Trape, C. Girmenia, L. Pagano., "Aspergillus niger infection in patients with haematological diseases: a report of eight cases.," Mycoses, vol. 47, pp. 163-167, 2004. 